\title{
Bulletin de I'APAD
}

\section{$L \cdot A \cdot P \cdot A \cdot D$}

7| 1994

Les sciences sociales et l'expertise en développement

(I)

\section{Le marché de l'expertise et la place du socio-anthropologue : le chercheur en sciences sociales en Afrique. Illustrations camerounaises}

Motaze Akam

\section{OpenEdition}

Journals

Édition électronique

URL : http://journals.openedition.org/apad/2293

DOI : 10.4000/apad.2293

ISSN : 1950-6929

Éditeur

LIT Verlag

Édition imprimée

Date de publication : 1 juillet 1994

Référence électronique

Motaze Akam, « Le marché de l'expertise et la place du socio-anthropologue : le chercheur en sciences sociales en Afrique. Illustrations camerounaises », Bulletin de l'APAD [En ligne], 7| 1994, mis en ligne le 13 décembre 2007, consulté le 08 septembre 2020. URL : http://journals.openedition.org/apad/2293 ; DOI : https://doi.org/10.4000/apad.2293

Ce document a été généré automatiquement le 8 septembre 2020.

Bulletin de l'APAD 


\title{
Le marché de l'expertise et la place du socio-anthropologue : le chercheur en sciences sociales en Afrique. Illustrations camerounaises
}

\author{
Motaze Akam
}

Introduction

1 Quelle position occupe le chercheur en sciences sociales au Cameroun? Au-delà des textes administratifs, le véritable statut du chercheur doit être saisi à travers les pratiques professionnelles au sein de son environnement institutionnel, et dans ses rapports avec la société globale qui permettent de le positionner par rapport au marché de l'expertise. L'expérience camerounaise illustre à cet effet un ensemble de textes institutionnalisant d'une part, la recherche en sciences sociales, et définissant, d'autre part, le statut de son chercheur. Malgré cette institutionnalisation et ce statut purement administratifs, la réalité est bien autre: les pratiques et problèmes de la recherche et les rapports chercheur-société exposent au grand jour la marginalisation dont le spécialiste des sciences sociales et la recherche sont victimes. Par conséquent, seule une redéfinition, voire une nette revalorisation de la profession de chercheur impliquant toutes les composantes qui structurent le marché de l'expertise en développement est en mesure de servir de solution à cette situation que connaissent le sociologue ou le socio-anthropologue et sa pratique scientifique.

Textes administratifs : institutionnalisation de la recherche en sciences sociales et statut du chercheur au Cameroun

2 L'histoire institutionnelle de la recherche au Cameroun est caractérisée par deux grandes périodes : la période sous administration française et celle indépendante. $\mathrm{Si}$ c'est notamment cette dernière qui intéresse ce propos, notons cependant quelques grands moments de la recherche durant la précédente. Ainsi, on retiendra, entre autres, la création de la société d'Etudes Camerounaises (SECAM) par un arrêté de l'administration française du 3 mars 1935. Créée en Afrique Occidentale Française 
(A.O.F.) par le gouverneur général Jules Brevie, une autre structure de recherche, l'Institut Français d'Afrique Noire (IFAN) cohabita de 1936 à 1938 avec la SECAM. Enfin, un autre arrêté créa l'Institut de Recherches Scientifiques du Cameroun (IRCAM) au mois de décembre 1949. Cet institut fut mis sous tutelle de l'ORSTOM alors Office de la Recherche Scientifique d'Outre-Mer et dont la naissance remonte à la loi du 11 octobre 1943. L'IRCAM fut au Cameroun, le principal représentant de l'ORSTOM ${ }^{1}$.

La période retenue ici se situant, naturellement, aux lendemains de l'indépendance du Cameroun est celle qui amena une recherche nationale et professionnelle. Par décret $n^{\circ}$ 62/DF/364, le nouveau gouvernement créa, le 2 octobre 1962, le Conseil de la Recherche Scientifique et Appliquée des Etudes et Enquêtes (CRSAEE). Parmi ses attributions, le CRSAEE, durant les missions internationales proposait des experts, chercheurs ou autres techniciens pour représenter le Cameroun dans les projets de rencontres scientifiques. Remplacera le CRSAEE, l'office National de la Recherche Scientifique et Technique (ONAREST) dont la création par la loi fédérale du 22 mai 1965 sera effective par le décret d'application $\mathrm{n}^{\circ} 74 / 588$ du 6 juin 1974. Les objectifs de l'ONAREST dépassent de loin ceux du CRSAEE. Il est chargé de l'orientation, de la coordination et du contrôle des activités de recherche à travers toute l'étendue nationale. Il doit, grâce à une recherche appliquée, promouvoir le développement économique et social. En outre, il supervise l'exécution des programmes de recherche, procède à la collecte des données dans les laboratoires à des fins d'une mise sur pied d'une documentation scientifique et de publication des résultats de recherche, assume la formation des chercheurs et techniciens et assure la liaison avec les corps scientifiques étrangers et internationaux.

C'est au sein de l'ONAREST que la recherche, en sciences sociales apparut avec deux organismes publics : l'Institut National de l'Education (INE) et l'Institut des Sciences Humaines (ISH). Mais les activités de l'INE vont se fondre dans celles de l'ISH qui survécut au décret $n^{\circ}$ 76/116 du 16 mars 1976 réorganisant l'ONAREST et réduisant ses neuf instituts à cinq. Un autre décret $n^{\circ}$ 76/121 du 16 mars 1976 portant spécifiquement réorganisation de l'ISH dote celui-ci de quatre centres de recherche dont la mission se résume à l'élaboration des programmes, la réalisation des recherches et le développement expérimental en vue de favoriser l'épanouissement du potentiel humain et de revaloriser l'élément humain dans le développement national, notamment :

- par la meilleure connaissance de la personnalité nationale, du patrimoine historique et culturel ;

- par la plus grande compréhension de l'environnement : humain, physique, géographique, économique, socio-culturel ;

- par l'étude des lois économiques et sociales, l'analyse des facteurs des données et des contraintes du développement ainsi que par la promotion expérimentale à travers les réalisations concrètes et contrôlées;

- par la diffusion des résultats de recherche, en liaison avec les services et organismes compétents.

L'avant-dernier grand moment de la réorganisation de la recherche en sciences sociales fut le 4 décembre 1979 où le décret $n^{\circ}$ 79/495 mit sur pied la Délégation Générale à la Recherche Scientifique et Technique (DGRST). Si les objectifs assignés à l'ISH restèrent intacts, le changement notable, sous la houlette de la DGRST et de la carrure de M. 
Vroumsia Tchinaye, son délégué, fut la promulgation, le 18 juillet 1980 , du décret $n^{\circ}$ $80 / 275$, portant statut des chercheurs.

L'avènement d'un statut des chercheurs marqua un événement décisif pour la profession du chercheur qui, jusque là, en était dépourvu. Ce décret est un document extrêmement important sur le plan juridico-administratif en tant qu'il garantit - non sans lacunes -les conditions et les modalités de l'exercice de la profession du chercheur au Cameroun. Toutes les préoccupations du chercheur s'y trouvent en termes de recrutement, évaluation, mise en stage ou en formation, changement de grade, primes de recherche et de technicité, etc. Ici, le chercheur peut être, soit un fonctionnaire détaché d'un ministère technique, soit un contractuel recruté selon les modalités prévues. Sa carrière passe par quatre grades: Attaché de recherche, Chargé de recherche, Maître de recherche, Directeur de recherche. Son changement de grade s'obtient au terme d'une évaluation scientifique de ses travaux.

7 L'érection de la DGRST en MESRES ${ }^{2}$, MESIRES ${ }^{3}$, MINREST ${ }^{4}$ connue ces dernières années n'a pratiquement rien apporté de neuf aux structures et chercheurs de l'ISH. Ce dernier par exemple, a toujours gardé ses caractères institutionnels liés à une administration publique à budget autonome, jusqu'à son dernier grand moment - triste celui-là marquant sa mort par un décret signé par le Président Biya, $n^{\circ}$ 91/416 du 18 octobre 1991 portant sa dissolution. Et en son chapitre 3, article 9, alinéa 1, le texte sus-évoqué dit simplement que : "Les modalités de transfert à l'Université de Yaoundé des activités jusque-là menées par l'ISH ainsi que de son personnel ayant le statut de chercheur professionnel font l'objet d'un texte particulier du Premier Ministre".

Jusqu'à présent, le "texte particulier" du Premier Ministre n'est toujours pas sorti. Mais le moins qu'on puisse dire est que, à travers ce décret présidentiel, l'institution universitaire prendra au Cameroun le relais de la recherche et des chercheurs en sciences sociales. Au terme de ce bref parcours juridico-administratif et institutionnel, il convient maintenant d'examiner les grands traits qui ont marqué les pratiques et problèmes de la recherche qui nous occupe ${ }^{5}$.

Recherche en sciences sociales au Cameroun : pratiques et problèmes

L'ISH a donc été, jusqu'au 18 octobre 1991, le cadre unique dans lequel se pratiquait une recherche professionnelle, c'est-à-dire que les chercheurs qui y exerçaient le faisaient à plein temps et ne s'occupaient pas en principe d'autres activités. Au vu des objectifs assignés à l'ISH, le type de recherche dont ses chercheurs exécutaient les programmes était la recherche-développement ou appliquée. Les programmes de recherche étaient issus des priorités gouvernementales en termes de besoins économiques et sociaux de développement selon les différents plans quinquennaux. La recherche était donc, dès le départ, orientée vers l'intervention. Partant des projets de développement bien précis, on demandait par exemple aux sociologues, anthropologues, économistes, etc., des investigations bien ponctuelles. Dans ce cadre, dès notre recrutement à l'ISH, notre formation de chercheur a été liée à une investigation sociologique qui devait servir de données de base au projet Centre-Nord qui nécessitait une étude des problèmes fonciers devant servir à l'expansion de la culture cotonnière dans le Nord du Cameroun. L'on peut être tenté de dire qu'une telle pratique de recherche s'apparentait déjà, à bien des égards, à un travail d'expertise.

De 1981 à 1985, période pendant laquelle des fonds substantiels ont été alloués à la recherche par l'Etat, les socio-anthropologues conscients de leur profession, motivés par le nouveau statut dont ils venaient d'être bénéficiaires ont produit une énorme 
"littérature grise" suite à diverses missions de terrain. Cette période que l'on peut taxer à juste titre de "période de vaches grasses", la "crise" étant encore ignorée du Cameroun, a malheureusement étalé les limites des chercheurs et des dirigeants de la recherche en matière d'une véritable politique scientifique concernant les sciences sociales. L'absence quasi totale d'une politique de valorisation a gangrené au sein des spécialistes des sciences humaines une lourde hypothèque ayant consacré le déficit total de leur existence scientifique et sociale jusqu'à aujourd'hui. Aussi, la valorisation est le point cardinal d'où partent tous les problèmes des sciences sociales et de leurs spécialistes au Cameroun. La valorisation a ainsi fonctionné comme paradigme ayant généré des problèmes qui lui sont inhérents à un double niveau et qui ont eu des conséquences multidirectionnelles aux plans scientifique et social.

11 Sur le plan scientifique, la valorisation a connu des problèmes liés à la fois à la publication des résultats de recherche, à leur diffusion, à l'animation et la vulgarisation scientifiques, et aux techniques d'évaluation même des travaux des chercheurs.

S'agissant des problèmes relatifs à la publication des résultats de recherche, ils se posaient sous un autre ordre, car se ne sont pas les structures de publication qui ont manqué à l'ISH. Ce qui leur a fait défaut et problème est qu'elles n'ont jamais connu de dynamisme à l'opposé des structures de l'administration coloniale connues avant. En effet, deux principales instances de publication existaient à l'ISH : la Revue Science et Technique, série Sciences Humaines et la collection Travaux et Documents de L'ISH. Si la première instance ne devait s'en tenir qu'à la publication des articles n'excédant pas trente pages, la seconde eut pour vocation de publier les travaux allant jusqu'à trois cents pages et au-delà. Etait déterminant au niveau des publications, le comité de lecture. La plupart de ses membres se plaignaient de ne pas bénéficier des quotas financiers de motivation, malgré les primes de recherche, de technicité et d'enseignement supérieur imbriquées dans les salaires des chercheurs et enseignants de rang magistral supposés le constituer. Les chercheurs envoyaient des travaux, selon la procédure en vigueur, et les services de la documentation, de la bibliothèque et des publications de l'ISH les acheminaient auprès des membres du comité, mais les avis attendus accusaient des retards de huit mois, un an ou ne venaient même jamais. Pour la Revue Science et Technique, sa périodicité trimestrielle devint semestrielle et cela ne changea rien. La collection Travaux et Documents supposée publier deux à trois livres chaque année connut un blocage total. Si on ajoute d'autres obstacles de nature purement structurelle (lenteurs administratives et absentéisme), il n'était plus étonnant que la Revue accusât des retards de plus de trois ans durant la décennie 80 ! Tandis que les Travaux et Documents ont fini carrément par ne plus exister. Comme corollaire, les problèmes de publication générèrent ceux de la diffusion. En effet de 1981 à 1990, quelques dix numéros de la Revue Science et Technique et dix-huit volumes des Travaux et Documents ont pu paraitre. Mais sur le plan national, on ne les trouvait dans aucune librairie. En règle générale, dans les grandes villes du Cameroun Yaoundé, Douala - il était extrêmement difficile d'accéder à quelques travaux publiés par l'ISH. A Yaoundé, grande ville universitaire et intellectuelle, pour les avoir, on devait nécessairement se rendre à la Direction de l'Institut. Evidemment, certaines universités et institutions de recherche américaines, françaises, hollandaises, belges etc. ayant signé des conventions en matière de coopération scientifique avec l'ISH recevaient notamment sa Revue, mais toujours est-il que la diffusion restreinte de ses quelques travaux a correspondu à une publication en hoquets. 
13 Aussi, la vulgarisation et l'animation scientifique ont-elles eu des difficultés énormes à prendre corps au sein de l'Institut et sur le plan strictement national. La vulgarisation et l'animation scientifiques auraient consisté à l'organisation des séminaires, conférences, colloques, symposii où utilisateurs des résultats de recherche, bailleurs de fonds et chercheurs auraient dialogué, discuté. Le dialogue et la discussion étant, dans cet ordre d'idées, les facteurs fondamentaux d'intégration d'un marché de l'expertise, mais lorsque la publication tâtonne et que la diffusion reste restrictive, la vulgarisation et l'animation scientifiques ne peuvent non plus contribuer à rendre vivants, les chercheurs et la recherche.

Ainsi, les chercheurs réellement travailleurs ne pouvaient rien faire que garder dans les tiroirs "la littérature grise" déjà évoquée. Ce qui créa d'énormes problèmes liés aux techniques de leur évaluation scientifique. Le statut des chercheurs évoqué a prévu l'évaluation des chercheurs sur la base de leurs travaux afin de pouvoir changer de grade. Autrement dit, les commissions scientifiques se devaient d'apprécier à leur juste valeur, les travaux réalisés par les chercheurs mais, paradoxalement, on exigeait que ces derniers présentent ces travaux publiés. Ceci causa une grande controverse et la controverse immobilisa chaque fois les évaluations. En ajoutant que celles-ci s'opérait à la tête du client, la plupart des chercheurs productifs et qui publiaient effectivement se trouvaient malheureusement bloqués à des grades, qui, au vu des textes régis par le statut des chercheurs, ne leur convenaient plus. Le laxisme de l'administration de la recherche et la complaisance dont les hauts décideurs politico-administratifs ont fait montre pour décanter un tel problème ont plutôt encouragé la frustration et la démobilisation de plusieurs chercheurs. Qu'on publie ou pas, tout le monde était tenu de rester au même grade !

Tous ces problèmes liés aux publications, à la diffusion des résultats de recherche, à la vulgarisation et à l'animation scientifiques, à l'évaluation même des chercheurs ont contribué à faire de la valorisation scientifique un non-lieu à l'ISH. Un protocole d'accord signé entre les structures de recherche et l'Université de Yaoundé en 1984 a semblé poser des bases d'une coopération scientifique entre celles-ci et celle-là.

Ce document a défini les modalités de circulation des chercheurs et enseignants dans les espaces universitaires et scientifiques. Encore en formation, les chercheurs pouvaient s'inscrire en thèse. Il a même été prévu la création des UER (Unités d'Etudes et Recherches) qui regrouperaient les enseignants et chercheurs de même discipline. Les chercheurs, soit en termes d'étudiants doctorants, soit en termes d'enseignants devaient poursuivre leurs travaux dans le cadre des structures universitaires dont la fonction aura alors été celle de leur valorisation par les soutenances et les publications. L'Université aura valorisé ainsi les résultats des travaux des chercheurs par des thèses soutenues ou leurs éventuelles publications. Mais un tel protocole d'accord n'a fonctionné qu'à sens unique, car seuls les enseignants venaient occuper les postes de responsabilité à l'ISH. Conséquence : de tels problèmes spécifiquement scientifiques, ont fait du chercheur Camerounais de l'ISH un être isolé, voire un illustre inconnu dans le domaine de son intervention, la recherche.

17 Sur le plan de la valorisation sociale, il convient d'examiner les problèmes inhérents aux rapports chercheurs/société. Il s'agit de voir la position qu'occupe le chercheur dans la société globale par rapport aux pouvoirs sociaux, publics, au pouvoir politique et au pouvoir même du chercheur qui est la connaissance scientifique et sa capacité de s'organiser. 

globale camerounaise atomisée. Ainsi institutions, religion, idéologies, formes d'organisation dites traditionnelles, partis politiques, syndicats, associations, constituent les pouvoirs sociaux à des échelles variées. Le rapport que le socio-anthropologue entretient avec les pouvoirs sociaux dans cette optique est essentiellement celui de sujet à objet (sans exclure que la recherche ou les sciences sociales elles-mêmes puissent aussi être objet d'étude du socio-anthropologue). Le chercheur étudie donc, dans ses investigations, ces phénomènes pour maîtriser la connaissance des milieux humains. Ces phénomènes sont comme on le verra plus loin, ceux qui lui confèrent le pouvoir scientifique et social - puisqu'en les analysant, il accède au savoir, à la connaissance scientifique, leitmotiv de tout développement. Son pouvoir social émane de la connaissance scientifique qui lui permet d'intervenir efficacement dans la société en matière de développement. Or, les pratiques et problèmes évoqués n'ont pas permis au chercheur camerounais d'assumer valablement ce rapport de connaissance. Aussi, à la crise institutionnelle vécue actuellement par le socio-anthropologue, nous pouvons ajouter la crise des connaissances sur les théories sociales camerounaises et des savoirs endogènes. moderne. Les pouvoirs publics peuvent aussi être objet d'étude des socio-anthropologues, mais selon les pratiques de la recherche au Cameroun, ils sont, notamment, les premiers organismes à bénéficier des investigations du chercheurs. Il faut compter ici les ministères techniques, les sociétés ou entreprises publiques et parapubliques, etc. Mais dans ces espaces, le sociologue 'Ou l'anthropologue est perçu conflictuellement par les techno-bureaucrates. Cette perception conflictuelle trouve son explication dans le fait que ces derniers ne voient en ce que font les chercheurs qu'une simple spéculation et prétexte même connaitre mieux qu'eux. Il y va ici de la perception même que ces techno-bureaucrates ont de la recherche : un travail inutile, puisque pour eux, tout le monde peut le réaliser. Et dès lors, il y a grève totale entre le socio-anthropologue et les pouvoirs publics qui, ironie du sort, ont été les premiers à lui faire des demandes d'intervention en besoins de développement. L'image du chercheur en sciences sociales dans l'administration camerounaise est ainsi celle de celui qui "bouffe" l'argent de l'Etat pour rien! L'échec des colonisations des terres au Nord Cameroun par exemple, et celui de la plupart des sociétés d'Etat, voire des organismes d'intervention en milieu rural dans le processus de développement s'expliquent, entre autres, par la non prise en compte des résultats des investigations des chercheurs nationaux dans ces domaines. La mentalité coloniale aidant, la bureaucratie locale préfère alors faire appel aux experts au mythe dépassé des grandes institutions internationales proposant comme solutions aux problèmes de développement, des modèles stéréotypés ne tenant compte d'aucune réalité historique, démographique, socio-économique, etc., des milieux à transformer. Le conflit chercheur/administrateur voue ainsi dans l'impasse, les rapports logiques devant exister entre socio-anthropologue et marché de l'expertise au Cameroun.

S'agissant du pouvoir politique, il se définit à un double niveau de relation sociale et de propriété. Il illustre au Cameroun les rapports d'un homme ou d'un groupe d'hommes à plusieurs autres garantissant à celui ou à ceux qui commandent la propriété des moyens de production. Le pouvoir politique est aussi objet d'étude et utilisateur des travaux des socio-anthropologues. Il est plus intéressant au Cameroun parce qu'il est 
l'instance suprême de décision. Car le Président de la République en personne est simultanément le Président du Conseil de l'Enseignement Supérieur et de la Recherche Scientifique. Au Cameroun, le pouvoir politique est l'instance suprême de marginalisation des chercheurs en sciences sociales. Tout semble indiquer aujourd'hui que la recherche, institutionnalisée, professionnalisée, autonomisée, n'a constitué qu'un dépotoir permettant de "caser" tout simplement les "grands cerveaux" à des fins $\mathrm{du}$ respect de l'ordre social. A l'ISH, on retrouvait les chercheurs de tout genre : les "virés de l'Université de Yaoundé", les "prêts-à-faire-valoir-leurs-droits-à-la-retraite", les "aigris", les médiocres, les dynamiques, les "chercheurs-fantômes" (ceux qui émargeaient tout simplement au budget de l'ISH tout en travaillant pour le compte du CENER ${ }^{6}$ ), etc. La logique de la recherche comme dépotoir ou ramassis initiée par l'ancien pouvoir pour faire taire les "intellectuels" par des gros salaires et autres avantages, sans effectivement tenir compte de leurs travaux, sans suivre leurs opérations de recherche, sans pénaliser les médiocres et encourager les excellents est encore brillamment entretenue par le pouvoir d'aujourd'hui. La crise actuelle de la recherche a permis de comprendre qu'au Cameroun, la recherche en sciences sociales et ses praticiens ne sont pour le pouvoir politique que des éléphants blancs ${ }^{7}$.

21 S'agissant enfin du pouvoir des chercheurs, celui-ci se définit au niveau d'un double objectif : maîtriser la connaissance scientifique et s'organiser. Organisation impliquant également un double aspect. Le premier est corrélatif au travail. En s'organisant en équipes de recherche pluridisciplinaires, on concrétise alors une recherche sans mur faisant acquérir plus de pouvoir aux chercheurs par de vastes connaissances sur leurs objets d'études. Mais, organisation aussi en un corps structuré capable de défendre les intérêts des chercheurs et de la recherche dans des sociétés où les droits sont bafoués au quotidien. Ce second aspect s'entend en termes syndicaux. Le désir d'accéder à la maitrise des connaissances scientifiques ne peut plus aller dans l'Afrique actuelle sans organisations syndicales autonomes des chercheurs: c'est là leur pouvoir. Mais au Cameroun, la plupart des chercheurs ignorent que le savoir dont ils sont détenteurs et les potentialités qu'ils ont à s'associer de façon autonome peuvent leur conférer un réel pouvoir dans la société globale. Le constat est donc que le rapport qu'ils entretiennent avec leur propre pouvoir est mitigé, traversé par une crise d'identité professionnelle. Contrairement au militaire, magistrat ou médecin, le chercheur est à la traîne de la société. Il est par là même encore, un illustre inconnu social.

Les problèmes de valorisation dans leur double dimension scientifique et sociale connus par le chercheur en sciences sociales au Cameroun permettent de dégager son statut réel. Inconnu sur le plan scientifique, à cause d'une gestion anti-scientifique de la recherche, rapport éconduit avec les pouvoirs sociaux, objet de sa connaissance, relations grevées ou dans l'impasse avec ses principaux demandeurs et utilisateurs des résultats, "casé" par le pouvoir politique quand il a domestiqué ou confisqué sa production intellectuelle, victime d'une crise d'identité professionnelle, le socio-anthropologue est alors une espèce de fantôme errant dans les sociétés qu'il doit pourtant aider à transformer, grâce au pouvoir scientifique. Marginalisé sur tous les plans ici examinés, son statut réel est qu'il n'a pas de statut. Et n'ayant pas de statut, tous ses rapports avec un marché de l'expertise sont brouillés, quasi inexistants. Ceci appelle, par conséquent, une redéfinition de sa profession.

Profession de chercheur, marché de l'expertise et ordre social 
23 D'après ce qui est dit dans les lignes précédentes, la problématique de l'expertise en général et la position du socio-anthropologue face au marché de l'expertise en particulier amènent à des considérations variées partant de l'expérience camerounaise.

24 Ainsi, par rapport à un marché de l'expertise, la profession de chercheur doit être priorisée. Cette priorisation est un processus devant intégrer les composantes essentielles du marché de l'expertise. Autrement dit, communautés de chercheurs, société, bailleurs de fonds, utilisateurs des résultats des recherches en sciences sociales et autres opérateurs de développement doivent constituer un ensemble où la professionnalisation du chercheur nécessite une intelligibilité de part et d'autre. L'on comprend dès lors que le problème n'est pas d'avoir des professionnels de l'expertise distincts des chercheurs, mais des professionnels de la recherche; en d'autres termes, de véritables chercheurs professionnels. Ceci pour la simple raison que l'expert véritable est avant tout chercheur, et le chercheur véritable est nécessairement expert.

Si le socio-anthropologue a des rapports brouillés avec le marché de l'expertise, ce n'est pas qu'il soit incapable d'y intervenir efficacement, encore moins qu'il soit incompétent ou totalement inconnu, il est tout simplement victime d'un préjugé défavorable par rapport à la société qui le méconnaît. Et c'est là où il nous faut axer toute réflexion pour voir comment arriver à une professionnalisation réelle du chercheur en dehors des cadres institutionnels purement administratifs, isolés, marginalisant la profession des socio-anthropologues. Pour ce faire, deux points nous paraissent cardinaux: œuvrer pour une redéfinition et une revalorisation de la profession du chercheur et intégrer la société dans les processus scientifiques. Qu'est-ce à dire?

S'agissant du premier point, les communautés - nationale et internationale - des chercheurs doivent mettre en œuvre une politique scientifique où la professionnalisation du métier de chercheur est la préoccupation primordiale. Car notre objectif global prioritaire est aujourd'hui, d'opérer une prise de conscience générale et une émule entretenue auprès de tous les partenaires concernés afin que le formidable potentiel de savoir des sciences sociales soit reconnu, mieux apprécié, mieux intégré tant du côté du Sud que du Nord ${ }^{8}$.

La professionnalisation a, sous-jacente, deux idées-forces : les considérations éthiques du métier de socio-anthropologue ; autrement dit, au-delà du respect et de la confiance mutuels entre nous, considérer désormais comme sacrée la profession de chercheur, qu'il soit du Sud ou du Nord. L'autre idée sous-jacente est la consolidation de la pluridisciplinarité pour concrétiser ce qui est convenu d'appeler aujourd'hui, recherche sans mur. Une telle conception de la recherche est à même de redéfinir et de valoriser la profession de chercheur, en l'occurrence, au Sud où en dehors des problèmes que nous avons recensés concernant le Cameroun, la crise et les programmes d'ajustement n'ont cessé d'aggraver les problèmes économiques, politiques, sociaux et financiers, rendant essentiellement précaire l'institution recherche. C'est l'occasion de saluer l'avènement de l'APAD. Née il y a quatre ans, nous espérons trouver à travers son partenariat euro-africain, la pluridisciplinarité qui se profile à travers les recherches anthropologiques sur le changement social et le développement qu'elle mène, la redéfinition et la revalorisation de notre profession pour un réel statut du chercheur en Afrique. La professionnalisation est donc avant tout, la propre affaire des chercheurs-spécialistes-experts que nous sommes. 

espaces sociaux, des conditions locales, des réseaux sociaux des principaux acteurs constituant les composantes qui la structurent. Et de là, son marché acquiert tout d'une société, il est lui même société. Et comme société, il est un lieu d'échanges et de négociations, de tensions et de dialogue, de conflits et de régulation, d'action et d'interactions : il doit donc être consensuel, s'il n'est pas intégré. Il doit reconnaître à chacun son statut, sa profession et son rôle véritable. Ce tout consensuel doit aider à la sécrétion des conditions de production des sciences sociales extrêmement rares dans le Sud. La professionnalisation dont nous parlons, expertise accumulée, pourra, dans un tel cadre, aider à la circulation efficace des socio-anthropologues dans le marché de l'expertise en développement entendu maintenant comme un certain ordre social à respecter.

Conclusion chercheurs dont l'institutionnalisation et le statut n'ont été consignés que sur du papier - à travers les textes administratifs. Les pratiques et problèmes de cette recherche ont évolué vers l'inexistence aujourd'hui de cette dernière. Les compétences nationales qui y exerçaient ont eu du mal à s'exprimer, à valoriser scientifiquement leurs travaux et à se faire valoriser socialement à cause de la gestion alimentaire dont 
cette recherche a été victime. Ceci a mitigé les rapports intéressants qu'il aurait dû entretenir avec la société, et partant le marché de l'expertise.

Nous avons dans ce cas les socio-anthropologues dont la profession a été ignorée et à la limite, bafouée par la société, même si eux-mêmes n'ont pas pu s'organiser pour défendre leurs intérêts scientifiques. Une telle situation du chercheur en Afrique amène plusieurs considérations dont, entre autres, la redéfinition et revalorisation de la profession hors des cadres purement institutionnels administratifs. Le véritable problème à résoudre est ici un processus engageant à des degrés variés, le marché de l'expertise en développement comme société où sociologues, anthropologues, économistes, juristes, historiens, démographes, etc., bailleurs de fonds, clientèles des sciences sociales, autres opérateurs de développement, etc., se doivent de mettre sur pied des stratégies consensuelles pour un construit d'ensemble. Pour ce qui est de notre part, nous restons optimistes qu'une politique scientifique fondée sur la professionnalisation de notre métier et une approche du marché de l'expertise comme société à construire impliquant les composantes essentielles des sciences comme filière (chercheurs et tous ceux qui sont intéressés par les résultats de recherche en sciences sociales) peut garantir des rapports efficaces entre socio-anthropologues et marché de l'expertise.

\section{BIBLIOGRAPHIE}

Boiral P., Lantéri J.-F., Olivier de Sardan J.-P. (dir.) 1985 : Paysans, experts et chercheurs en Afrique Noire, CIFACE, Karthala, Paris, 224 p.

Chatelin Y. et Arvanitis R. : 1984, "Pratiques et politiques scientifiques", Actes du Colloque des 6 et 7 février 1984 ORSTOM, $192 \mathrm{p}$.

Forje J.W. 1987, Science, technology and development policy in Cameroon, Centre for action-oriented research on African development, 309 p.

Gast M. et Panoff M. (dir.) : 1986, L'accès au terrain en pays étranger et outre-mer, l'Harmattan, Paris, $303 \mathrm{p}$.

Motaze Akam : (à paraître), Recherche en sciences sociales, politique et faillite du développement au Cameroun, (sociologie d'une crise), l'Harmattan, Paris, 266 p.

Tedga J.P.M. : 1988, L'enseignement supérieur en Afrique noire francophone : la catastrophe ?, PUSAF/ L'Harmattan, Paris, 224 p.

"Découverte et innovation scientifiques au service du Tiers-Monde", Revue Tiers-Monde XX, (78), 1979, pp. 225-438.

"L'épistémologie des sciences sociales. Statut, valeurs et institutionnalisation, Revue internationale des sciences sociales XXXVI, (4), 1984, pp. 597-766. 


\section{NOTES}

1.Le sigle ORSTOM signifie aujourd'hui : Institut Français de Recherche Scientifique pour le Développement en Coopération.

2.Ministère de l'Enseignement Supérieur et de la Recherche Scientifique.

3.Ministère de l'Enseignement Supérieur de l'Informatique et de la Recherche Scientifique.

4.Ministère de la Recherche Scientifique et Technique.

5.Pour plus de détails en ce qui concerne l'histoire institutionnelle de la recherche en sciences sociales évoquée ici, voir : Motaze Akam : Recherche en sciences sociales, politique et faillite du développement au Cameroun (sociologie d'une crise), L'Harmattan, Paris (à paraître).

6.CENER, Centre National d'Études et Recherches n'utilisant que la police politique du régime en place.

7.Rappelons ici que malgré les graves problèmes ayant, amené la dissolution de l'ISH, le Conseil de l'Enseignement Supérieur et de la Recherche Scientifique n'a connu sa dernière convocation que sous Ahmadou Ahidjo!

8.Une telle idée est exprimée depuis 1986 par une communauté d'ethnologues et anthropologues occidentaux : elle garde ici toute son actualité. Voir à propos : M. Gast et $M$. Panoff (sous la direction de) L'accès au terrain en pays étranger et outre-mer, l'Harmattan, Paris, 1986, 303 pp., p. 247.

9.R Arvanitis "Pratiques et politiques scientifiques", Actes du Forum des 6 et 7 février 1984 organisé par Yvon Chatelin et Rigas Arvanitis, ORSTOM, Paris, 192 pp., p.40.

10.Edmond Burke III "L'institutionnalisation de la Sociologie en France : sa portée sociale et politique" in L'épistémologie des Sciences sociales. Statut scientifique et institutionnalisation, Revue Internationale des Sciences Sociales, XXXVI, (4), 1984, p. 677-690, p. 678. 